\title{
Flebotomíneos em área de transmissão de leishmaniose tegumentar na região norte do Estado do Paraná - Brasil: Variação Sazonal e Atividade Noturna*
}

\author{
Phlebotomines in an area of the transmission of American cutaneous \\ leishmaniasis in the north of Parana State, Brazil: Seasonal Variation and \\ Nocturnal Activity
}

Ueslei Teodoro**, Vicente La Salvia Filho**, Edson Mauricio de Lima**, Roberto Palma Spinosa***, Orlando Carlos Barbosa***, Maria Eugênia Moreira Costa Ferreira****, Thaís Gomes Verzignassi Silveira**

\begin{abstract}
TEODORO, U. et al. Flebotomíneos em área de transmissão de leishmaniose tegumentar na região norte do Estado do Paraná - Brasil: Variação Sazonal e Atividade Notuma. Rev. Saúde Pública, 27: 190-4, 1993. No período de dois anos de capturas de flebotomíneos com armadilha luminosa de Shannon, às margens de uma mata residual alterada, na fazenda Palmital, Município de Terra Boa, Estado do Paraná, Brasil, observou-se que a densidade populacional desses insetos diminuiu sensivelmente, quando comparada com resultados anteriores, no mesmo local. As espécies predominantes, Lutzomyia whitmani, Lutzomyia migonei, Lutzomyia intermedius e Lutzomyia fischeri tiveram atividade das 18 às $6 \mathrm{~h}$ e o pico horário de frequência variou de acordo com cada espécie. As espécies citadas mostraram maior densidade nos meses de verão e outono. Concluem-se que as alterações introduzidas no ambiente podem ter resultado em mudanças no comportamento dos flebotomíneos.
\end{abstract}

Descritores: Psychodidae. Ecologia de vetores. Leishmaniose mucocutânea, transmissão.

\section{Introdução}

O modo clássico de infecção por protozoários do gênero Leishmania, dos complexos "braziliensis" e "amazonensis", está na dependência do contato dos indivíduos com o ambiente florestal (Gomes \& Galati ${ }^{7}, 1989$ ).

No Norte do Estado do Paraná, onde as alteraçōes ambientais vêm ocorrendo desde a década de 40 , e os parasitas identificados têm sido Leishmania (Viannia) braziliensis e Leishmania (Leishmania) amazonensis (Silveira e col. ${ }^{12}, 1990$ ), a leishmaniose tegumentar é endêmica e apresenta fortes indícios de que a infecção humana está acontecendo no ambiente extraflorestal, em nível domiciliar e peridomiciliar.

\footnotetext{
* Financiado pelo Núcleo de Estudos de Saúde Coletiva Paraná, PR - Brasil

* Departamento de Análises Clínicas da Universidade Estadual de Maringá - Maringá, PR - Brasil

*** Fundaçāo Nacional de Saúde - Londrina, PR - Brasil

**** Departamento de Geografia da Universidade Estadual de Maringá, PR - Brasil
}

Separatas/Reprints: U. Teodoro - Av. Colombo, 3690 - 87020900 - Maringá, PR - Brasil
Assim, a ocorrência de leshmaniose tegumentar endêmica e a escassez de informaçōes sobre flebotomineos motivaram a realização do presente trabalho que visou a complementar dados relativos a variação sazonal e a atividade notuma desses dípteros no Norte do Estado do Paraná.

\section{Material e Método}

As capturas de flebotomíneos foram executadas numa área da fazenda Palmital (Fig. 1), de propriedade da Companhia Melhoramentos Norte do Paraná, no Município de Terra Boa, de maio de 1988 a abril de 1990 , em região já descrita ${ }^{13}$. As coletas de flebotomíneos foram feitas no mesmo local onde se trabalhou anteriormente, ou seja, às margens de mata residual situada a $10 \mathrm{~m}$ de uma colônia de casas, agora desabitadas, e sendo usadas como depósitos de adubo. Utilizou-se armaditha de Shannon com auxílio de lampião, de 300 velas, colocado no centro da mesma. As capturas foram feitas mensalmente, por 2 indivíduos, usando-se tubos de vidro com algodão embebido com clorofórmio. Foram realizadas 24 capturas, das 18:00 às 6:00 horas. 


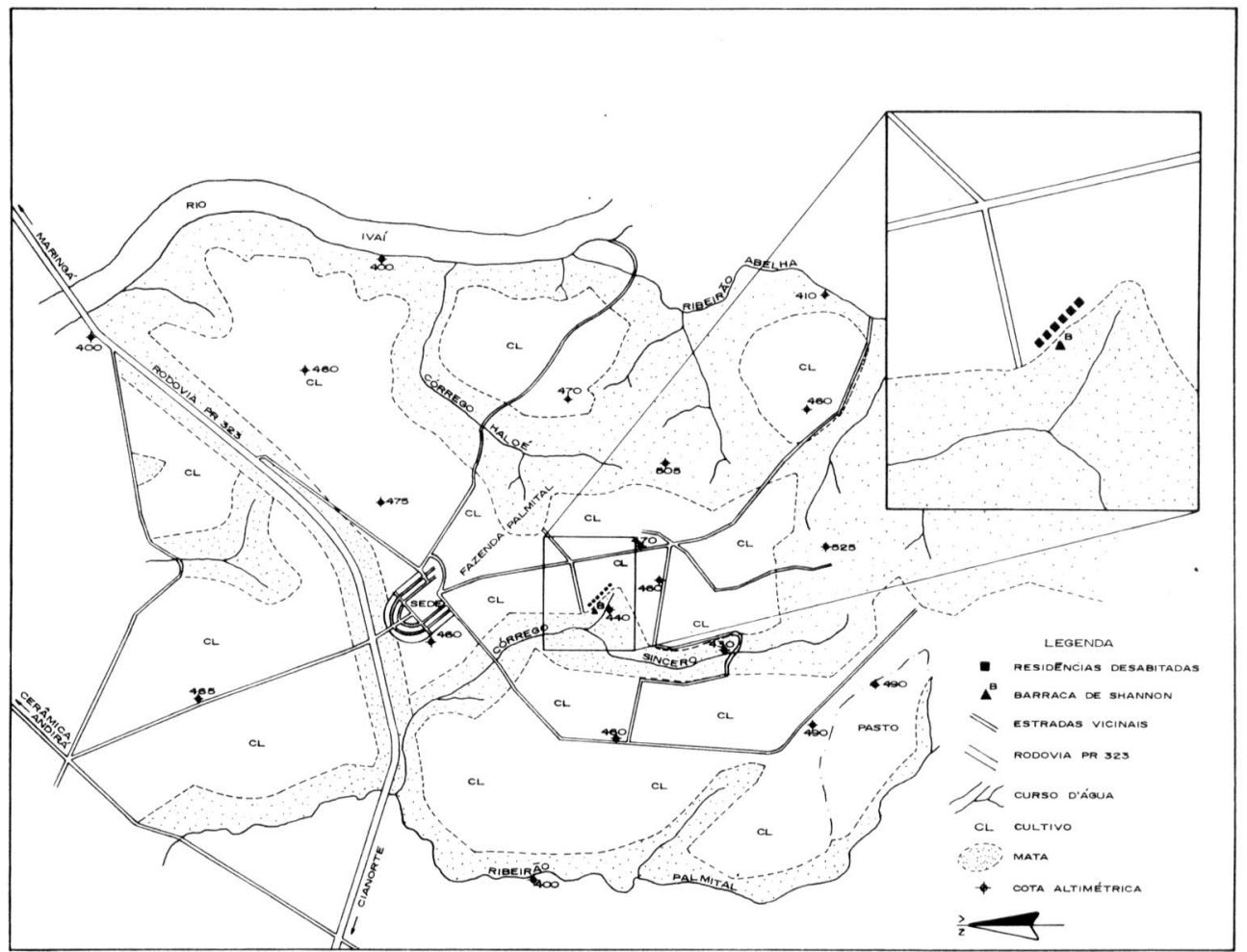

Figura 1. Local de capturas de flebotomíneos, com armadilha de Shannon, na fazenda Palmital, Município de Terra Boa, Estado do Paraná, de maio de 1988 a abril de 1990.

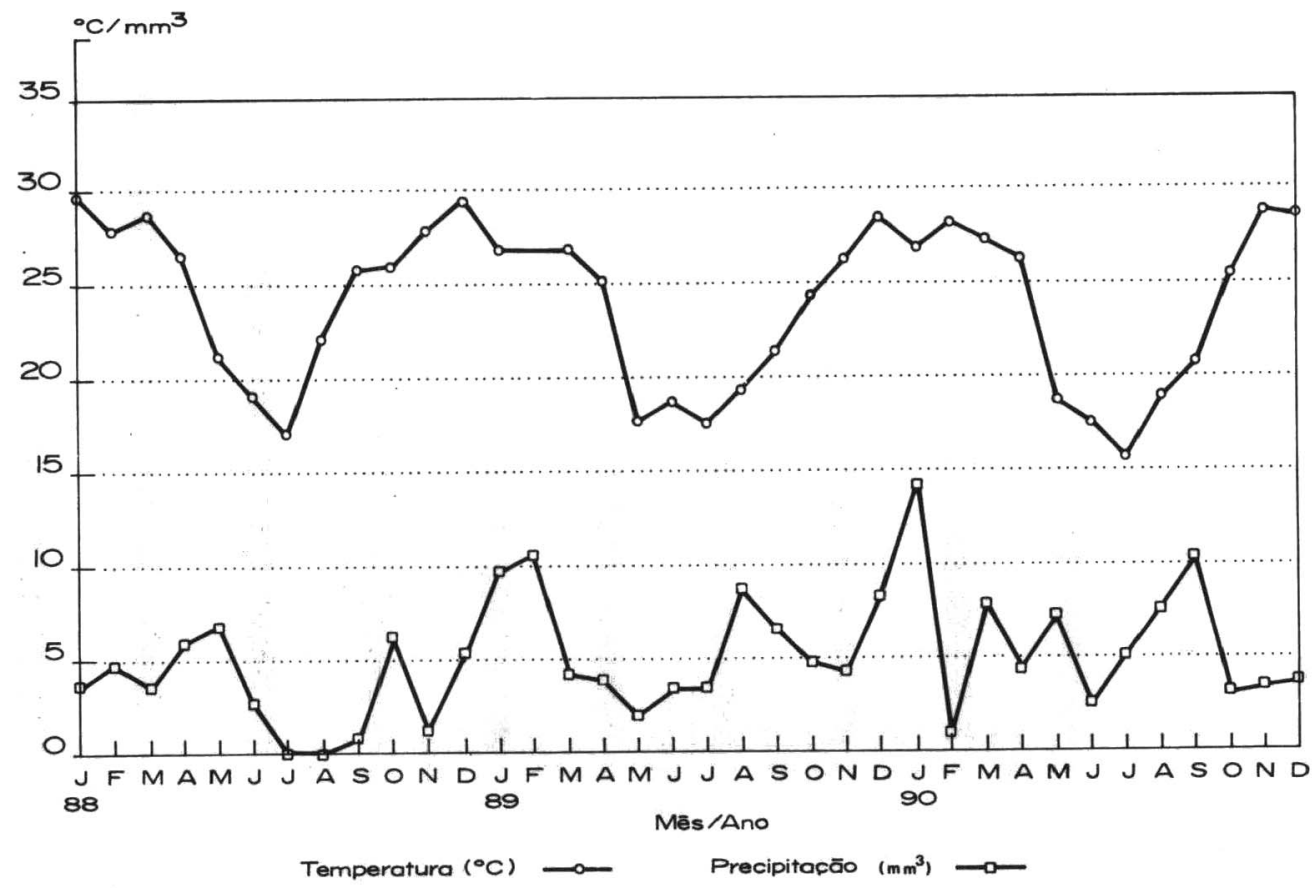

Figura 2. Médias mensais de temperaturas e precipitaçōes pluviométricas nos anos de 1988 a 1990, na fazenda Palmital, Município de Terra Boa, Paraná, Brasil. 
No presente trabalho adotou-se a nomenclatura proposta por Martins e col. ${ }^{11}$ (1978).

Os dados de temperatura e precipitação pluviométrica foram coletados na fazenda Palmital e fornecidos pela Companhia Melhoramentos Norte do Paraná (Fig. 2).

\section{Resultados}

Foram identificados 4.548 flebotomíneos, distribuídos quase todos em 13 espécies, sendo 953 machos e 3.595 fêmeas, conforme mostra a Tabela. Observou-se a prevalência de $L$. whitmani $(68,0 \%)$, seguido por $L$. migonei $(18,2 \%), L$. intermedius $(6,0 \%)$ e L. fischeri $(2,8 \%)$ (Tabela).

Verifica-se na Figura 3 que as espécies citadas foram capturadas das 18 às $6 \mathrm{~h}$, havendo aumento gradual da atividade de $L$. withmani até às $5 \mathrm{~h}$, com flutuações das outras 3 espécies isoladamente. $L$. migonei mostrou maior atividade entre 1 e $3 \mathrm{~h} \mathrm{e} L$. intermedius entre 22 e $24 \mathrm{~h}$, enquanto $L$. fischeri foi mais ativo das 22 às 23 horas e das 0 à 1 hora.

Na Figura 4 vê-se que a densidade populacional de $L$. withmani flutuou durante os 2 anos, havendo densidades elevadas nos meses de maio e julho de
Tabela. Flebotomíneos capturados nas margens de mata residual na fazenda Palmital, Municipio de Terra Boa, de maio de 1988 a abril de 1990.

\begin{tabular}{lrrrr}
\hline Espéie & \multicolumn{1}{c}{ O } & \multicolumn{1}{c}{ Total } & $\%$ \\
\hline Lutzomyia whitmani & 452 & 2.642 & 3.094 & 68,0 \\
Lutzomyia migonei & 344 & 485 & 829 & 18,2 \\
Lutzomyia intermedia & 86 & 186 & 272 & 6,0 \\
Lutzomyia fischeri & 33 & 95 & 128 & 2,8 \\
Lutzomyia shannoni & 3 & 68 & 71 & 1,6 \\
Brumptomyia brumpti & 29 & 38 & 67 & 1,5 \\
Lutzomyiamisionenis & - & 32 & 32 & 0,7 \\
Lutzomyia amarali & - & 21 & 21 & 0,5 \\
Lutzomyia pessoai & 1 & 12 & 13 & 0,3 \\
Lutzomyia monticola & 2 & 10 & 12 & 0,3 \\
Lutzomyia cortelezzii & - & 4 & 4 & - \\
Brumptomyianitzulescui & 3 & - & 3 & - \\
Lutzomyia lanei & - & 1 & 1 & - \\
Brumptomyia sp & - & 1 & 1 & - \\
\hline Total & 953 & 3.595 & 4.548 & \\
\hline
\end{tabular}

1988, outubro de 1989 e janeiro de 1990 , mas os picos ocorreram nos meses de abril e maio de 1989, e março de 1990. Nota-se que as densidades de L. migonei foram altas em setembro de 1988, abril, maio e agosto de 1989, com maior densidade em janeiro de 1989 (Fig. 4). L. intermedius e L. fischeri apresentaram pequenas densidades no decorrer de 2

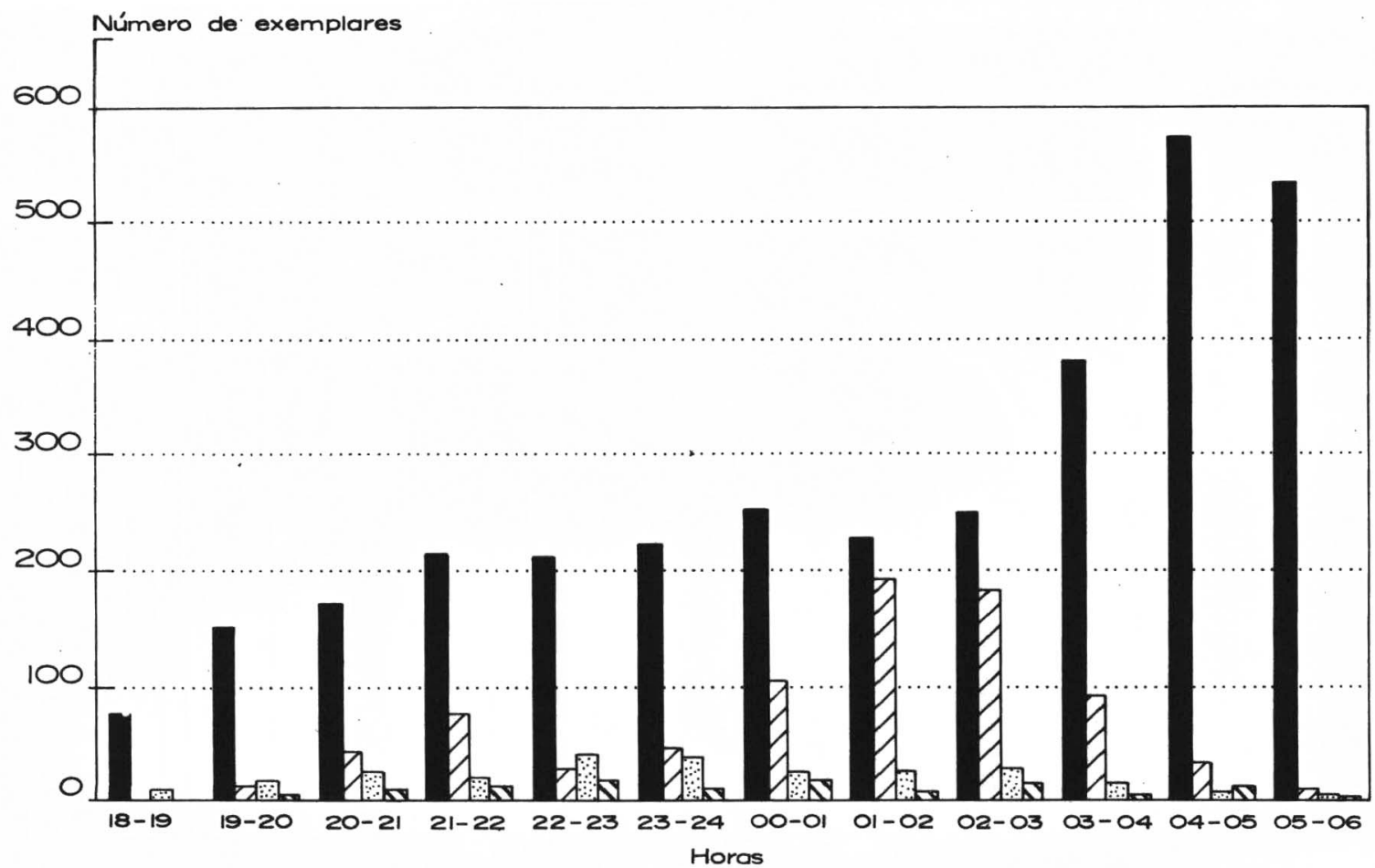

P. whitmani

B. migonei

$\because \because$ P intermedius

DV P. fischeri

Figura 3. Média horária de atividade dos flebotomineos mais freqüentes e de importância vetorial entre 18 e horas na fazenda Palmital, Município de Terra Boa, Estado do Paraná, de maio de 1988 a abril de 1990. 


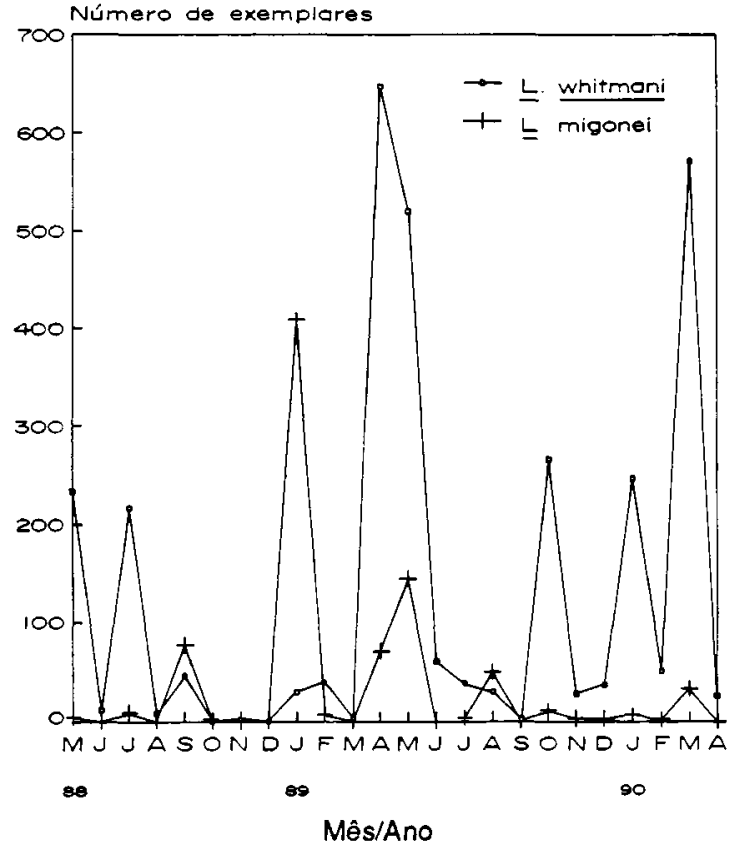

Figura 4. Variação sazonal de Lutzomyia whitmani e Lutzomyia migoneina fazenda Palmital, Município de Terra Boa, Estado do Paraná, de maio de 1988 a abril de 1990.

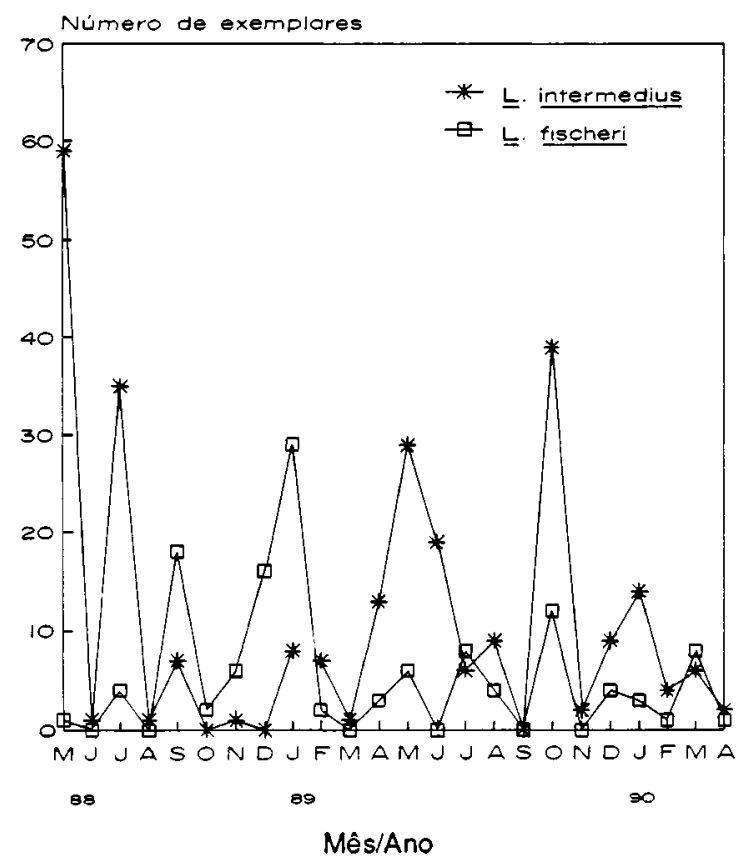

Figura 5. Variação sazonal de Lutzomyia intermedius e Lutzomyia fisheri na fazenda Palmital, Municipio de Terra Boa, Estado do Paraná, de maio de 1988 a abril de 1990.

anos, sendo o maior pico do primeiro, em maio de 1988, e do segundo, em janeiro de 1989 (Fig. 5).

\section{Discussão}

Às 13 espécies descritas anteriormente ${ }^{13}$ na fazenda Palmital, acrescente-se a presença de $L$. la$n e i$, assinalada na região por Aguiar e col. ${ }^{1}$ (1989).

Confirmou-se que $L$. withmani, $L$. migonei, $L$. intermedius e $L$. fischeri aqui são predominantes, cabendo esclarecer que, em relação ao mencionado estudo $^{13}, L$. migonei passou a ocupar a segunda posição como espécie prevalente, ao invés de $L$. intermedius. A alternância de posição dominante foi observada anteriormente por Forattini ${ }^{4}$ (1960). Gomes \& Galati ${ }^{6}$ (1977), em área florestal primária, no Município de Londrina, PR, e Aguiar e col. ${ }^{1}$ (1989), no peridomicílio, na margem de floresta e no seu interior, no Município de Jussara, PR, verificaram o predominio de $L$. withmani em todos os ambientes, exceto no peridomicílio, onde prevaleceu $L$. intermedius. Esses autores mostraram tambérr que $L$. intermedius não esteve presente no interior de matas, L. fischeri em geral preponderou na mata, além de apresentar altas densidades em todos os ambientes, ao lado de $L$. migonei, que estava presente em pequenas densidades.

Os trabalhos anteriores ${ }^{1,3,6,13}$, no Estado do Paraná, não esclareceram sobre a atividade de flebotomíneos durante todo o período noturno. Na Figura 3 observa-se o aumento constante da atividade de $L$. withmani, que atingiu o maior pico entre $4 \mathrm{e}$ $5 \mathrm{~h}$, coincidindo com a tendência antes verifica$\mathrm{da}^{13}$. O mesmo não ocorreu com L. migonei que ar:teriormente ${ }^{13}$ manteve atividade c-escente até $2 \lesssim \mathrm{h}$, decaindo daí por diante, enquanto que nesta investigação apresentou flutuações, atingindo o máximo de atividade entre 1 e $3 \mathrm{~h}$, para diminuir em seguida. Em trabalho anterior ${ }^{13} L$. intermedius e $L$. fischeri apresentaram crescimento contínuo de atividade até uma hora, observando-se agora oscilações durante todo o periodo roturno.

Tem-se conhecimento que a maioria das espécies de flebotomineos tende a aumentar a densidade nos meses mais quentes e úmidos ${ }^{6,10}$, apesar da existência de espécies que se mostraram mais densa nas épocas secas e frias ${ }^{3,8}$. Comparando-se as Figuras 2 e 4 nota-se que $L$. withmani prevaleceu no verão e outono, mas apresentou densidades elevadas no inverno e na primavera. Em relação a $L$. migonei, a maior densidade foi observada no verão 'Figuras 2 e 4 ), a de $L$. intermedius no outono (Figuras 2 e 5) e L. fischeri no verão (Figuras 2 e 5), não diferindo de informações obtidas anteriormente ${ }^{13}$, no mesmo local.

As ações antrópicas sobre a natureza podem influir decisivamente na disponibilidade de hospedeiros $^{2,9}$ e Gomes $^{5}$ (1985) sugeriu que a habitação de má qualidade, em condições de abrigar animais domésticos e sinantrópicos, favorece a formação 
de biocenose artificial da leishmaniose tegumentar, "da qual o homem é parte integrante". Os 16.946 exemplares de flebotomíneos coletados por Teodoro e col. ${ }^{13}$ (1991), em 24 capturas, durante um ano, num total de $168 \mathrm{~h}$, no mesmo local, superam em muito os 4.548 agora coletados, também em 24 h, durante 2 anos, e em 288 horas. Esta alteração na densidade populacional de flebotomíneos talvez possa ser explicada pelo abandono das habitações com a conseqüente ausência de seres humanos e de animais domésticos (cães, gatos, suínos e galinhas), que possivelmente serviam como fontes sanguíneas para esses dípteros. Além disso, não se viu mais lixo doméstico e outros resíduos que eram atirados na mata, no local onde foram realizadas as capturas.

\section{Agradecimentos}

À diretoria da Companhia Melhoramentos Norte do Paraná, com sede no Município de Cianorte, e aos administradores da fazenda Palmital pelas facilidades que ofereceram para a realização do presente trabalho; ao Sr. Carmelindo Colato, do Departamento de Engenharia Civil da Universidade Estadual de Maringá, pelos serviços de desenho.

TEODORO, U. et al. [Phlebotomines in an area of the transmission of American cutaneous leishmaniasis in the north of Parana State, Brazil: Seasonal Variation and Nocturnal Activity. Rev. Saúde Pública, 27: 190-4, 1993. In two years of phlebotomine captures in a modified forest an appreciable decrease in the size of this insect population has been observed when compared to previous data from the same locality. The captures with the Shannon light trap were made in the outlying forest of Palmital farm in Terra Boa county, Parana State, Brazil. The predominant species were Lutzomyia withmani, Lutzomyia migonei, Lutzomyia intermedius e Lutzomyia fischeri. These insects were active from 6 p.m. to 6 a.m. and the period of greatest activity varied according to the species. These species' densities were higher in the summer and autum months. Environmental modifications may have influenced the observed changes in phlebotomine behavior.

Keywords: Psychodidae. Ecology, vectors. Leishmaniasis mucocutanea/transmission.

\section{Referénclas Bibllográficas}

1. AGUIAR, G.M. de et al. Ecologia de flebótomos em recente foco ativo de leishmaniose tegumentar no Norte do Estado do Paraná (Diptera, Psychodidae, Phlebotominae). Mem. Inst. Oswaldo Cruz, 84 (Supl. 4): 7-8, 1989.

2. ARAGÃO, M.B. Sobre o comportamento de alguns insetos hematofagos. Arq. Biol. Tecnol., 18: 3-23, 1975.

3. CONSOLIM, J. et al. Flebótomos da área do reservatónio da hidrelélrica de Itaipu, Estado do Paraná, Brasil (Diptera, Psychodidae). Cad. Saúde Pública, 6: 74-85, 1990.

4. FORATTINI, O.P. Novas observaçס̃es sobre a biologia de flebótomos em condiçōes naturais (Diptera, Psychodidae). Arq. Fac. Hig. S. Paulo, 25: 209-15, 1960.

5. GOMES, A. de C. Aspectos epidemiologicos sobre a transmissão da leishmaniose tegumentar na Região do Vale do Ribeira, Estado de São Paulo, Brasil. São Paulo, 1985 [Tese de Livre-docência - Faculdade de Saúde Pública da USP].

6. GOMES, A. de C. \& GALATI, E.A.B. Flebotomíneos de Londrina, Paraná (Brasil) e observações ecológicas sobre algumas espécies. Rev. Saúde Pública, 11: 284-87, 1977.

7. GOMES, A. de C. \& GALATI, E.A.B. Aspectos ecológicos da leishmaniose tegumentar americana. 7. Capacidade vetorial flebotomínea em ambiente florestal primário do sistema da Serra do Mar, Região do Vale do Ribeira, Estado de São Paulo, Brasil. Rev. Saúde Pública, 28: 136-42, 1989.

8. GOMES, A. de C. et al. Aspectos ecológicos da leishmaniose tegumentar americana. 2. Ecótopos artificiais como abrigo de $P s$. intermedius e observaçōes sobre alimentação e reprodução sob influência de fatores físicos naturais. Rev. Saúde Pública, 16: 149-59, 1982.

9. GOMES, A. de C. et al. Aspectos ecológicos da leishmaniose tegumentar americana. 3. Observações naturais sobre o ritmo diário de atividade de $P$ s. intermedius sm ambiente florestal e extraflorestal. Rev. Saúde Pública, 17: 23-30, 1983.

10. LE PONT, F. \& PAJOT, F.X. La leismaniase en Guyane Française. 1. Étude de l'ecologie et du taux infection naturelle du vecteur Lutzomyia umbratilis Ward \& Fraiha, 1977 en saison séche. Cah. OR.S.T.O M. Sér. Ent. Méd. Parasit. 18: 369-82, 1980.

11. MARTINS, A.V. et al. American sand flies (Diptera: Psy. chodidae, Phlebolominae). Rio de Janeiro, Academia Brasileira de Ciências, 1978.

12. SILVEIRA, T.G.V. et al. An autochthonous case of cutaneous leishmaniasis caused by Leishmania (Leishmania) amazonensis Lainson \& Shaw, 1972 from the North of Parana State, Brazil. Mem. Inst. Oswaldo Cruz, 85: 475-6, 1990.

13. TEODORO, U. et al. Leishmaniose tegumentar americana: flebotomíneos de área de transmissão no Norte do $\mathrm{Pa}$ raná - Brasil. Rev. Saúde Püblica, 25: 129-33, 1991.

Recebido para publicação em 1.9.1992 Aprovado para publicaçăo em 27.5.1993 